\title{
Channel Characteristics in Tunnels: FDTD Simulations and Measurement
}

\author{
L.A.R. Ramirez, F.J.V. Hasselmann, \\ CETUC - Centro de Estudos em Telecomunicações - Pontifícia Universidade Católica do Rio de Janeiro \\ Rua Marquês de São Vicente, 225, 22451-900, Rio de Janeiro, RJ, Brazil, \\ \{luis,flavio\}@cetuc.puc-rio.br \\ Y.P. Zhang \\ Nanyang Technological University, School of Electrical and Electronic Engineering, Singapore, Singapore \\ eypzhang@ntu.edu.sg
}

\begin{abstract}
This paper presents the results of measurements and simulations of the characteristics of $900 \mathrm{MHz}$ band radio propagation channels in a tunnel environment. The simulations were made using the FDTD method (with companion UPML) and measurements made use of the swept frequency technique. Another method, the metaheuristic Simulated Annealing, was implemented for estimating the values of characteristic parameters of materials. The FDTD code was reformulated for use with CUDA with the objective of decreasing program running time.
\end{abstract}

Index Terms - Tunnel, CUDA, FDTD, Simulated Annealing.

\section{INTRODUCTION}

A special type of indoor environment, tunnels are relevant for systems serving both roads and railways as well as in urban subway services. Leaky feed cables have been customarily used inside tunnels with the communication being provided by stringing wires over the length of the tunnel or by placing repeaters at intervals, or both. These methods are expensive to install and inconvenient, need regular maintenance, could be unreliable in disaster situations and do not allow an in-tunnel mobile user to transmit. The use of discrete antennas inside tunnels can avoid the above shortcomings, with the possible advantage of needing no prior infra-structure in peer-to-peer wireless communications.

The Finite-Difference Time-Domain (FDTD) method [1] is implemented with companion of Uniaxial Perfect Matched Layers (UPML) [2] to access the power delay profile and channel related parameters of a subway tunnel in Hong Kong, China, for a wide band UHF range centered at 900 $\mathrm{MHz}$ [3], [4]. Simulated results are compared with measurement data, validating the channel characterization. Equipment comprised a network analyzer, a sweep oscillator and discone antennas for both the transmitter and receiver ends as described in Section IV, while modelling techniques and the implementation of simulation are reviewed in section II and III, respectively.

\section{Modelling}

\section{A. Scenario modelling}

The scenario in Fig. 1.a, corresponding to the second of three sections of a Hong Kong subway tunnel, has a rectangular cross section with height and width $3.43 \mathrm{~m}$ and $2.6 \mathrm{~m}$, respectively, central 
section $107.7 \mathrm{~m}$ long, walls in reinforced concrete (relative permittivity $\varepsilon_{\mathrm{r}}=3.28$ and conductivity $\sigma=$ $0.05 \mathrm{~S} / \mathrm{m}$, approximate values calculated according to the technique in B), with antenna positions depicted in Fig.1.b
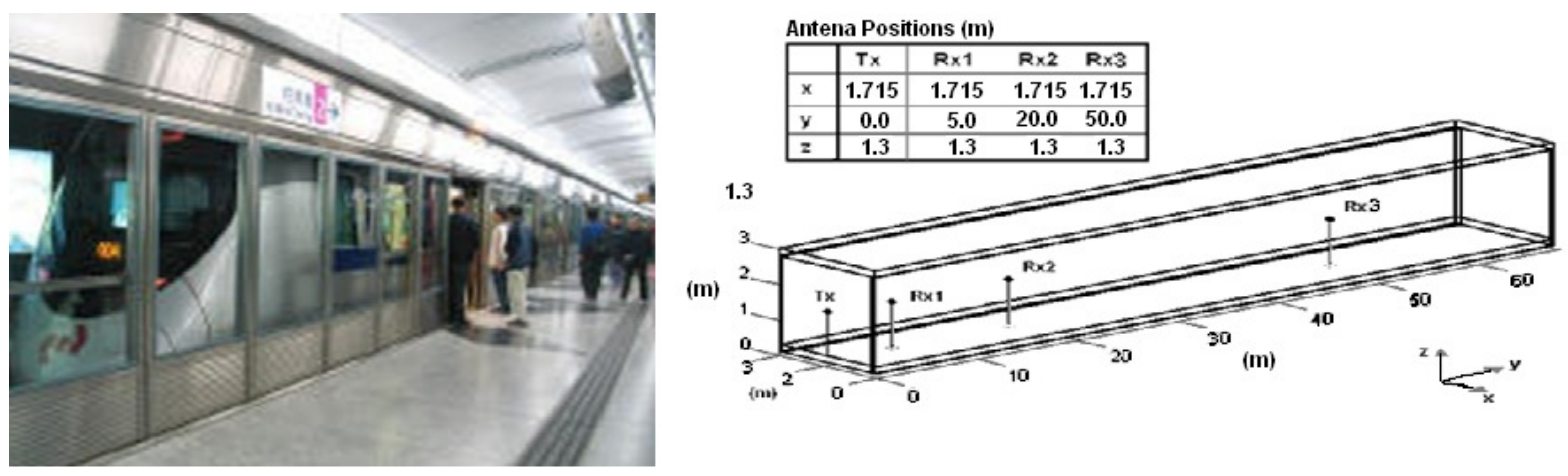

Fig. 1. Hong Kong subway tunnel (a) photo (b) structure used in the simulation.

\section{B. Simulated Annealing Technique: Practical Implementation for Characteristic Parameters Estimation.}

Lack of knowledge of characteristic parameters of walls materials made it necessary the use of a meta-heuristic technique, called "simulated annealing", in order to minimize the difference between estimated and measured values. The concept of the method is based on the manner in which liquids freeze or metals recrystalize in the process of annealing, thereby transforming a poor, unordered solution into a highly optimized, desirable one [5][6]. This process of reducing the mismatch is called calibration and it consists in extracting relevant multipath components (MPCs), for instance once reflected paths, simultaneously from the model and measurement. Afterwards, the simulated annealing is performed to optimize the material parameters. The calibration was performed using the measurements for LOS case Tx-Rx1 in Fig.1. Fig. 3 displays the obtained (before and after calibration) and measured power delay profile (PDP). As expected, the calibrated PDP shows a better match with the measured PDP for Tx-Rx1. The values encountered were used for the other cases considered in this work.

Details of the implementation are described in the following paragraphs. In the process of annealing used, the initial material properties were defined using known tabulated values for reinforced concrete $\left(\varepsilon_{\mathrm{r}}=4.95\right.$ and conductivity $\left.\sigma=0.01 \mathrm{~S} / \mathrm{m}\right)$ available in the literature[7].

As the relation between the power taps and material parameters is a non linear combinatorial relationship, this technique provides the general optimal solution by simultaneously changing the dielectric constant and conductivity of concrete parameters with a changing step at each range of iterations, for relevant multipath components(MPC).

The algorithm considers a subset of $\mathrm{M}$ measurements with $\mathrm{P}_{\mathrm{i}}$ power taps from each measurement $i$. The objective function, defined as the root mean square error (RMSE) between the measured and the predicted tap powers from all $\mathrm{M}$ measurements, is optimized to return a measure of the energy state 
or value of the present minimum, computed at each iteration $k=i . j$ as

$$
\operatorname{RMSE}_{k}=\frac{1}{M} \sum_{i=1}^{M} \frac{1}{P_{i}} \sqrt{\sum_{j=1}^{P_{j}}\left[\left(a_{i j}\right)_{\text {pred }}^{2}-\left(a_{i j}\right)_{\text {meas }}^{2}\right]_{k}^{2}}
$$

where the parameters $\left(a_{i j}\right)_{\text {pred }}^{2}$ and $\left(a_{i j}\right)_{\text {meas }}^{2}$ denote the predicted and measured power of the $j$-th MPC from the $i$-th measurement.

In the SA algorithm, random steps in the parameter space are performed using a control parameter $\mathrm{T}$ which is decremented as the algorithm progresses. A step is always accepted if the objective function is lowered, and it is sometimes accepted with a certain, decreasing probability, if an uphill step is taken. Its decreasing probability $\exp \left[-\Delta_{\mathrm{RMSE}} / \mathrm{T}\right]$ is a function of the variables $\left(\mathrm{RMSE}_{\mathrm{i}}\right.$, $\mathrm{RMSE}_{\mathrm{i}-1}$ and $\mathrm{T}$ ), where $\Delta_{\mathrm{RMSE}}$ is the difference between the actual RMSE $\mathrm{i}_{\mathrm{i}}$ and its neighbor RMSEi ${ }_{-1}$. This scheme allows the algorithm to escape from local minima. By decreasing the step, it computes the best value at each iteration as depicted in Fig. 2 with 1764 iterations for the convergence of the simulated annealing algorithm when minimizing RMSE. The objective function evolves from $7 \mathrm{~dB}$ to an optimal value of $0.3 \mathrm{~dB}$ for which the material parameters of the database are changed.

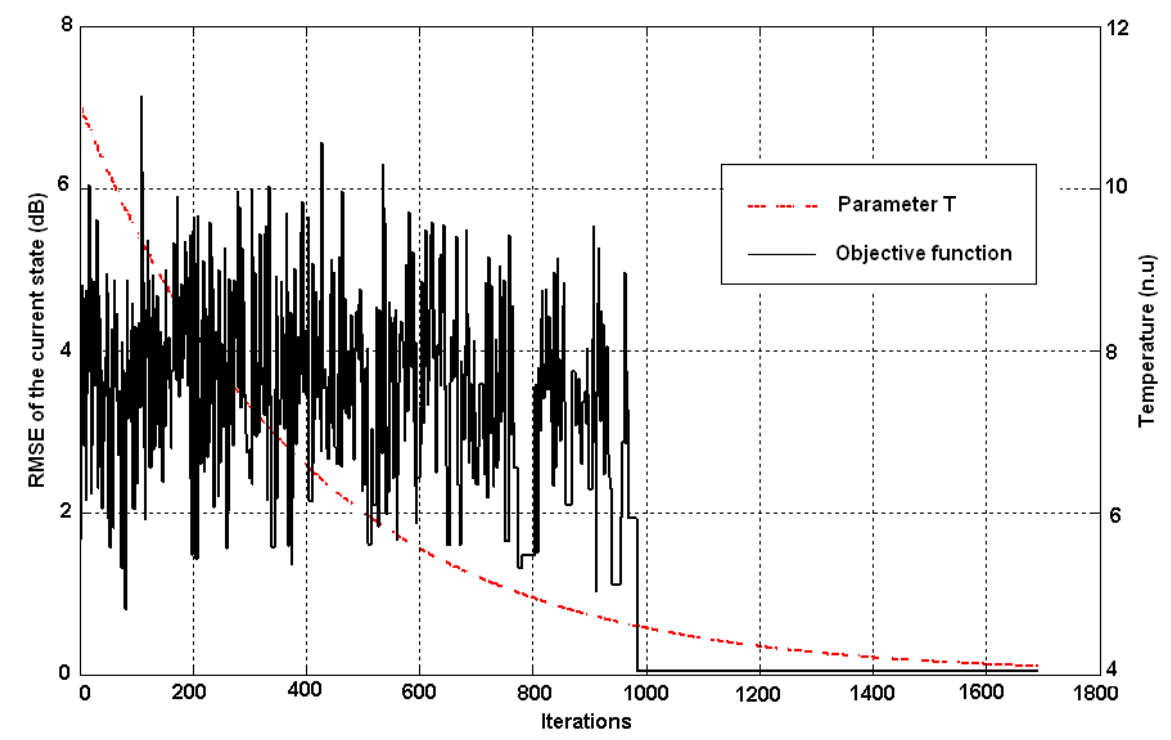

Fig. 2. Evolution of the RMSE of the FDTD prediction when choosing the material parameters using simulated annealing for an objective function variation within $42 \times 42$ iterations. The parameter $\mathrm{T}$ is expressed in natural units.

The algorithm stops after a certain number of steps NS if there is no significant change in the objective function. After termination, the final configuration is taken as the solution and a file containing the optimized parameters is produced. This optimized parameters can then be used by the 3D FDTD program. 


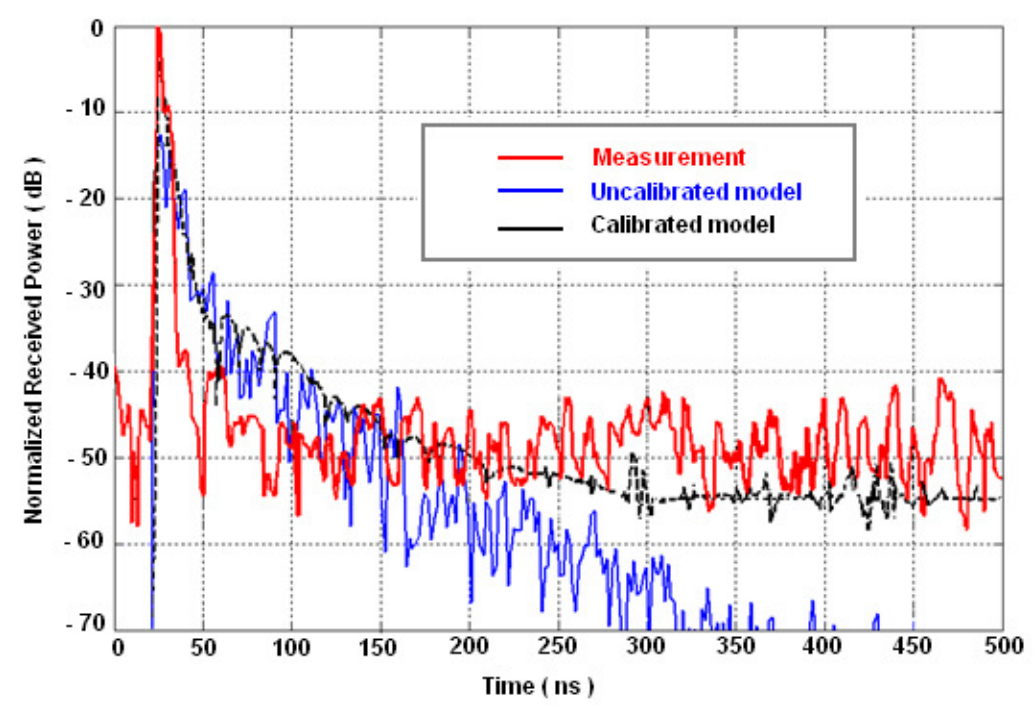

Fig. 3. Power delay profile comparison (measurement, uncalibrated and calibrated method).

\section{Antenna modelling}

The transmitter (Tx) and receiver antennas (Rx1, Rx2) in Fig. 1 are discones fabricated for the 900 MHz band, operating along wide bandwidths (>400 MHz). The Tx antenna, shown in Fig. 4.a, was inserted in the FDTD grid and components of electric field tangential to (metallic) elements considered null, with excitation as in (2).

The generated driving signal in (2) has a central frequency $\left(f_{c}\right)$ of $900 \mathrm{MHz}$ over a band $\left(f_{b}\right)$ of 450 $\mathrm{MHz}$ and $\mathrm{t}_{0}=5 \mathrm{~ns}$ denotes the initial delay; signal (normalized) amplitude and its frequency contents are illustrated in Figs. 5.

$$
\mathrm{z}(\mathrm{t})=\mathrm{E}_{0} \sin \left[2 \pi \mathrm{f}_{\mathrm{c}}\left(\mathrm{t}-\mathrm{t}_{0}\right)\right] \exp \left[-\left(2 \pi \mathrm{f}_{\mathrm{b}}\left(\mathrm{t}-\mathrm{t}_{0}\right)\right)^{2}\right]
$$
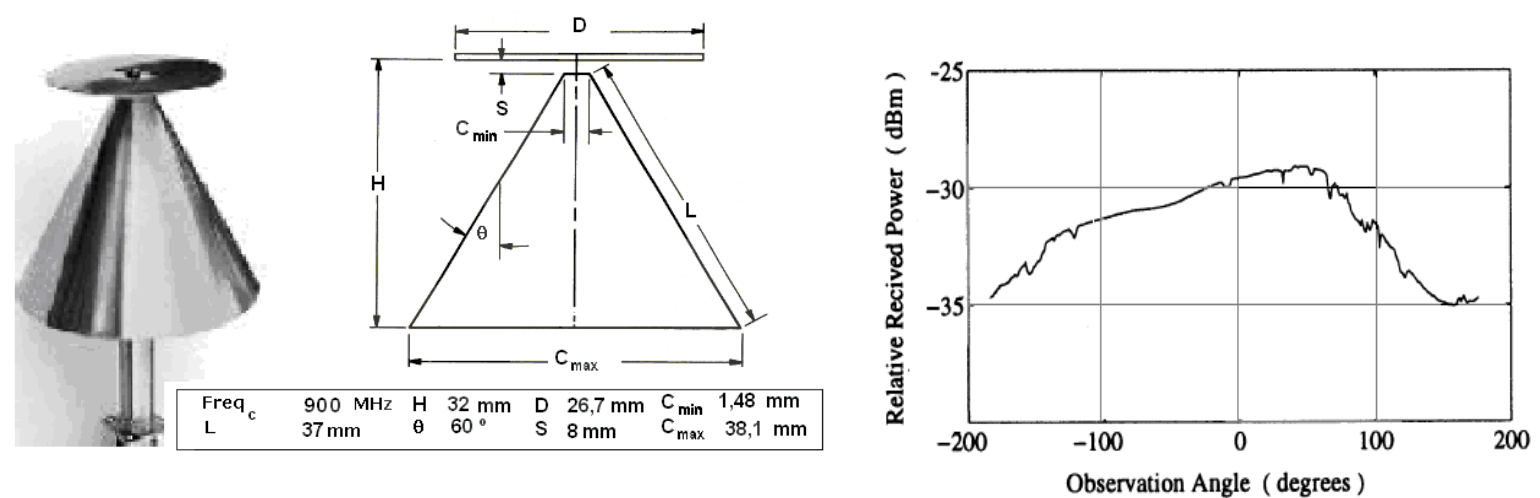

Fig.4.(a) Dimensions of transmitter and receiver antennas for $900 \mathrm{MHz}$. (b) Measured horizontal plane radiation pattern of discone antennas at $900 \mathrm{MHz}$.

Fig. 4.(b) illustrates their measured co-polarized horizontal plane radiation patterns at $900 \mathrm{MHz}$.

\section{FDTD IMPLEMENTATION}

As for the implementation of the FDTD method for the most critical configuration, the discretization of the computational domain followed an uniform grid with spatial step $\Delta x=\lambda / 10$, 
which, for a working frequency of $900 \mathrm{MHz}$ (wavelength $\lambda=0.3331 \mathrm{~m}$ ), corresponds to $\Delta \mathrm{x}=0.0333$ $\mathrm{m}$. Numerical dispersion was thereby avoided and, by proper choice of the temporal step $(\Delta t=0.0577$ ns), calculated according to Courant criteria [1] and used in central difference approximation of Maxwell equations, numerical stability was assured. Also, for the truncation of the domain, 7 UPML layers were implemented accordingly [2].
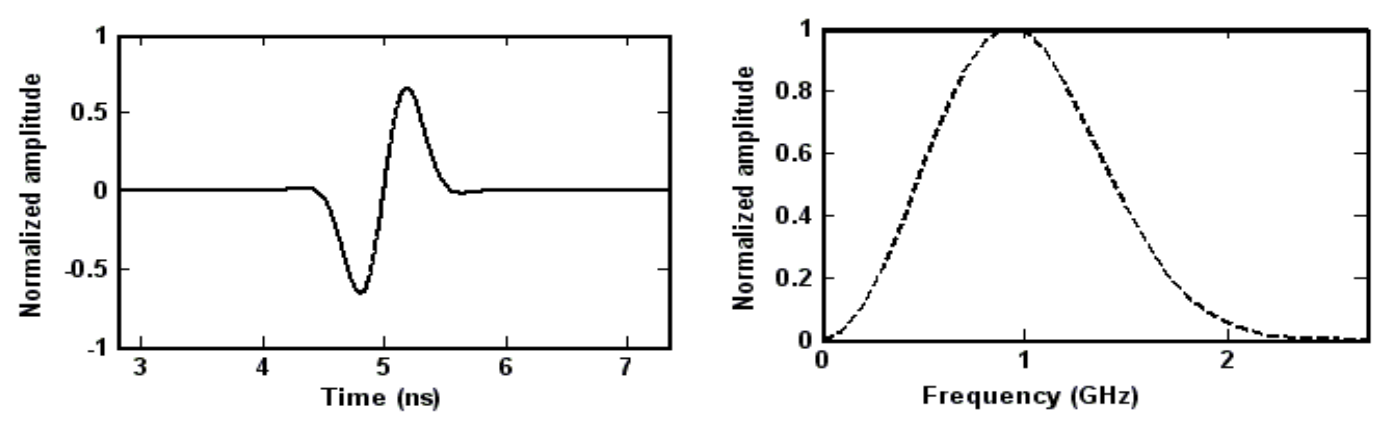

Fig.5. Pulse excitation of Tx antenna and its frequency spectrum.

\section{A. CPU implementation}

For the scenario $(60.0 \times 3.43 \times 2.6 \mathrm{~m})$ scenario at hand and a spatial step of $0.0333 \mathrm{~m}$, a total of 16251 FDTD cells are represented, each cell associated to six (electric and magnetic) field values as well as to six flags indicative of the type of material present at each spot. Also, field values are 4 bytes real numbers while flag lengths are 1 byte each, implying a memory need of 30 bytes per cell and a total memory requirement of circa 8.366 GBytes to address the present problem, which was perfectly handled by available 24 GBytes (RAM) processors. With the time step used, however, 17321x106 iterations were necessary to address the $500 \mathrm{~ns}$ duration of the simulations displayed above, rendering approximately 5 hours of computer time. In order to remedy this problem, the original code in $\mathrm{C}$ language was translated to CUDA (Compute Unified Device Architecture), a parallel computing architecture that use $\mathrm{C}$ with Nvidia extensions[8].

\section{B. GPU implementation}

Recent developments in graphics hardware acceleration provide operations that can be applied to dramatically speed up FDTD simulations and the economy of scale gained by 3D graphics cards due to the burgeoning video-game/entertainment industry provides a considerable cost/performance advantage. Graphics processing units (GPUs) are very fast, very cheap, and continue to be enriched at rates that outstrip those of general purpose CPUs. GPUs are constructed in a superscalar fashion. A GPU is implemented as an aggregation of multiple so-called multiprocessors, which consist of a number of SIMD ALUs (Single Instruction Multiple Data Arithmetic Logic Unit). A single ALU is called processor. According to the SIMD concept, every processor within a multiprocessor must execute the same instruction at the same time, only the data may vary[9]. The software model of CUDA offers the GPU as data-parallel coprocessor to the CPU. CUDA allows the programming of 
GPUs for parallel computation with minimum graphics knowledge, the framework CUDA providing to the programmer a minimal extension to use functions named kernel to call a process a number of times concurrently. In the CUDA model, being each data processed by an individual thread, each thread runs a distinct process or program. Threads are organized inside blocks, and blocks are in turn grouped inside grids. The kernel is called using a global declaration and the number of threads and blocks. Each process runs on a thread, and each thread has a number for its identification[8]. Each multiprocessor has a small shared memory that can be accessed from each of its processors, and there is a large global memory space common to all the multiprocessors. Shared memory is very fast and is usually used for caching data from global memory. Both shared and global memory can be accessed by any thread for reading and writing operations without restrictions[8].

These multiple levels of memory hierarchy are quite different from the common CPU memory/cache model. The CUDA memory hierarchy is composed of a large on-board global memory which is used as main storage for the computational data and for synchronization with the host memory. Unfortunately, the global memory is very slow and not automatically cached. To fully utilize the available memory bandwidth between the global memory and the GPU cores, data has to be accessed consecutively. To alleviate the high latencies of the global memory, a shared memory can be used. Shared memory is explicitly managed by the kernel and special care has to be taken when it is accessed. When the same shared memory banks are accessed by multiple threads at the same time, a memory access conflicts will occur and the readings to the same memory bank will be serialized[9].

A general-purpose computing on GPUs so-called GPGPU applications follows the steps:

1. Define and reserve memory for CPU and GPU data structures.

2. Copy data from CPU to GPU global memory

3. Define numThreads and numBlocks variables

4. Execute CUDA procedure for each thread

5. Copy data results from GPU to CPU

6. Free GPU data structures

The initial CUDA FDTD version of the code was implemented to work on GPU Quadro FX 1800 series with clock rate: $1400 \mathrm{MHz}$, multiprocessors: 8, threads per block: 512, global memory: 767.688 $\mathrm{MB}$, memory shared per block: $16 \mathrm{~KB}$. The $(\mathrm{x}, \mathrm{y}, \mathrm{z}) \mathrm{E}$ and $\mathrm{H}$ field components were stored on the device memory as 32 bit floating point variables. A texture memory was used to store, as a pointer stream, the material types in the model space. In both of these cases, the 3D volume was flattened into 2D and was accessed via an algorithm based on $3 \mathrm{D}$ to $2 \mathrm{D}$ address translation. This allows the entire 3D space to be updated in one render pass and also avoids potential "read after write" data corruption. The material type pointer stream was used for material property lookups stored in textures. E and $\mathrm{H}$ scattered field update calculations were converted to fragment programs, taking the $\mathrm{E}$ and $\mathrm{H}$ fields values stored in textures as inputs. Between each time step, input and output memories on the device are swapped providing a feedback loop and avoiding the performance penalty of pushing data across the system bus. Performance achieved with this version GPU was 10 times more fast that if compared 
to the CPU version one, it is believed that this should improve in upcoming versions of the program due to ongoing code optimization processes.

\section{MEASUREMENTS}

The wideband experimental setup is shown in Fig. 6 [3], [4]. The system consists of an HP 8350B sweep oscillator, $8510 \mathrm{C}$ network analyzer, and 8517A -parameter test set. Port 1 was connected to the transmit antenna through a $10-\mathrm{m}$ coaxial cable. The power outputted to the transmit antenna was 15 $\mathrm{dBm}$. The transmitted sweep frequency waves were captured by the receive antenna connected to port 2 through another $50-\mathrm{m}$ cable. The measured data of wideband propagation characteristics was stored onto a floppy disk of the network analyzer for subsequent analysis.

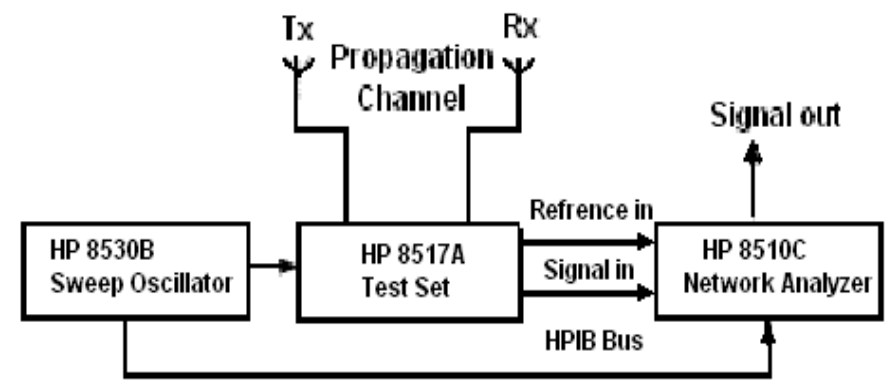

Fig. 6. The wideband experiment setup.

\section{COMPARASION BETWEEN SIMULATED AND MEASURED DATA}

Three situations were considered. In the line-of-sight (LOS) one (Figure 1), Rx1 was located very near $(5 \mathrm{~m}) \mathrm{Tx}$ and $\mathrm{Rx} 2$ was placed in the middle of the tunnel (antennas 50m apart). In the NLOS situations, the receiver antenna $\mathrm{Rx} 3$ was separated $20 \mathrm{~m}$ from the transmitter one and the path was obstructed by a vehicle modelled by a perfectly conducting parallelepiped of dimensions $1.0 \mathrm{~m} \mathrm{x}$ $1.35 \mathrm{~m} \times 0.45 \mathrm{~m}$ placed above the ground and located $10 \mathrm{~m}$ away from Tx.

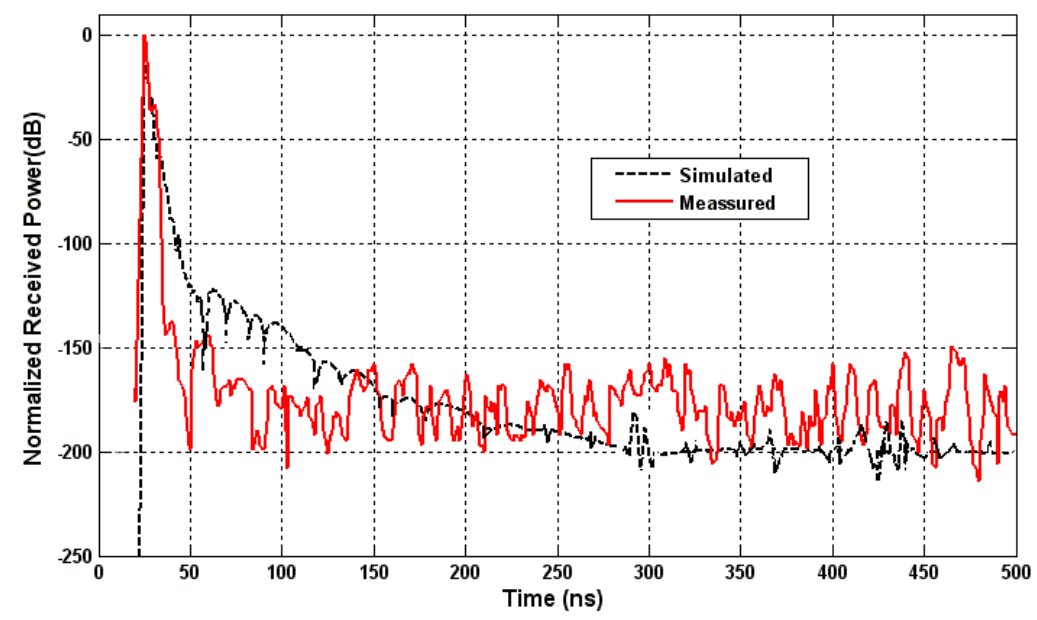

Fig. 7. Simulated (dashed line) and measured (continuous line) averaged $900 \mathrm{MHz}$ vertically polarized power delay profile (LOS). The distance between antennas is $5 \mathrm{~m}$.

Figure 7 illustrates the comparison between simulated and measured averaged $900 \mathrm{MHz}$ vertically 
polarized power delay profiles for a $5 \mathrm{~m}$ antenna separation. The first signal to reach the receiver, along the direct ray, is depicted at about 26ns, with previous contributions corresponding to noise. Deviations between measurement and simulations, thereafter, are credited to probe resolution which fails to take into account relevant reflected ray contributions for short distances between transmitter and receiver antennas.

Figure 8 show the comparison between averaged $900 \mathrm{MHz}$ vertically polarized power delay profiles for a $50 \mathrm{~m}$ antenna separation, which permits the probe resolutions to yield measured data followed more closely by simulations. Also, as expected, the main contribution following a direct propagation path, detected at about $167 \mathrm{~ns}$, is observed to be substantially stronger than those reaching the receiver via reflected paths with partial energy carried by the delayed waves being inevitably absorbed by the tunnel walls.

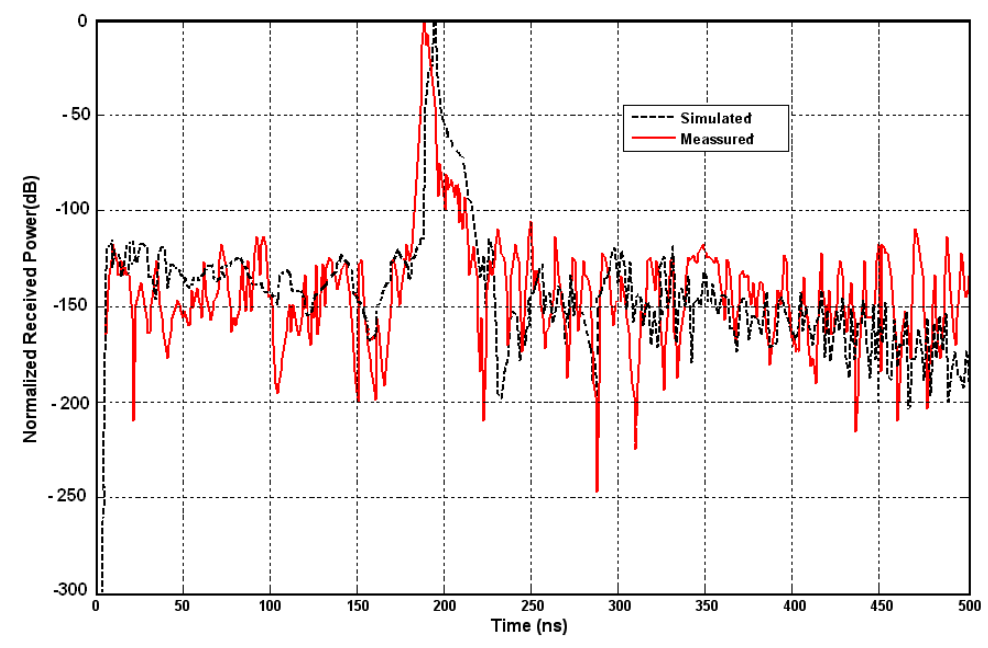

Fig. 8. Simulated (dashed line) and measured (continuous line) averaged $900 \mathrm{MHz}$ vertically polarized power delay profile (LOS) The distance between antennas is $50 \mathrm{~m}$.

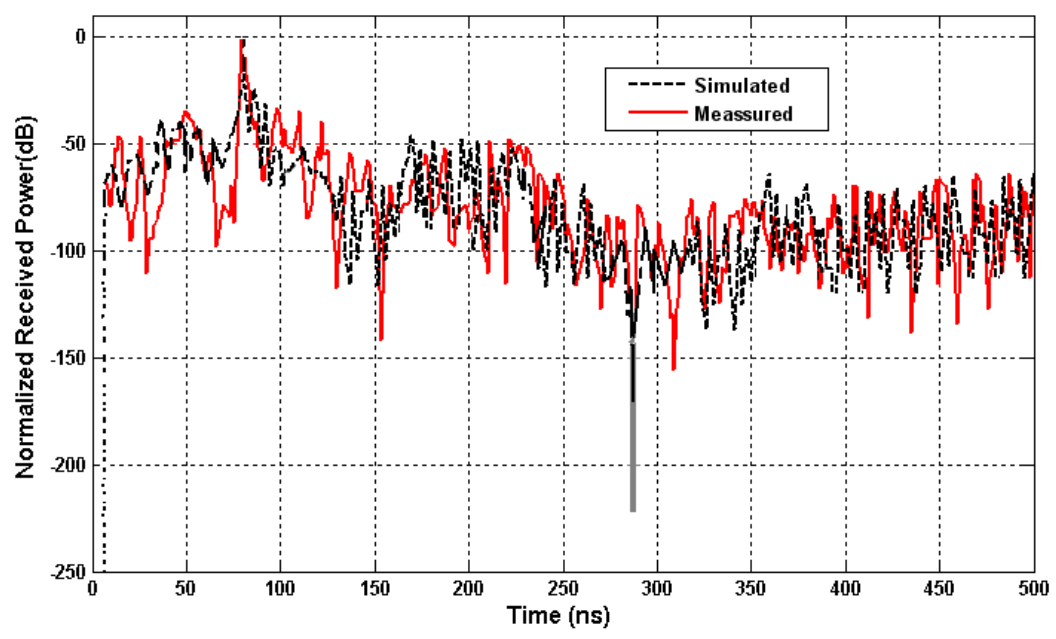

Fig. 9. Simulated (dashed line) and measured (continuous line) averaged $900 \mathrm{MHz}$ vertically polarized power delay profile (NLOS). The distance between antennas is $20 \mathrm{~m}$ with the path obstructed by a vehicle located $10 \mathrm{~m}$ away from the transmitter antenna.

Figure 9 shows the comparison between averaged $900 \mathrm{MHz}$ vertically polarized power-delay 
profile for a $20 \mathrm{~m}$ antenna separation with the line-of-sight path obstructed by a vehicle located $10 \mathrm{~m}$ away from the transmitter antenna. The direct signal reaches the receiver at $52 \mathrm{~ns}$ while the delayed (reflected) ones become stronger thereon, recovering maximum (line-of-sight) field intensity at $76 \mathrm{~ns}$. At about $200 \mathrm{~ns}$, scattering by the vehicle is observed to play its role. Again, equipment resolution is due to account for occasional deviations between interference curves.

\section{CONCLUSION}

In this work, a comparasion between (FDTD) simulated and measured results for the averaged power delay profile along a subway tunnel of simple cross section and finite conductivity walls, for both empty and partially filled situations, was presented. Although a good agreement between results has been observed, two important aspects of the simulations were revealed and explored. The determination of characteristic parameters of materials from the knowledge of measured data was addressed by the simulated annealing technique, which may yet be generalized and its implementation optimized. Also, excessive computing times, typical of this category of numerical simulations, were shown to be alleviated via the CUDA parallel programming route and further investigation and implementations are likely to improve its efficiency.

Work in progress explores the application of the techniques employed herein to tunnels of more general cross sections and more complex fillings, aiming at fully understanding the propagation of radiowaves in tunnels.

Future work will address the analytical description of mode guiding in tunnels in order to investigate coverage characteristics along extended frequency bands. Modal analyses can then be converted into ray-optical ones via appropriated Poisson Sums to access the localized tracking of field contributions to specific observation regions and, through Fourier inversions, the corresponding evolutions in time. In this way, a physical transparency may be added to FDTD results such as these presented herein, therefore contributing to its interpretation and desired validation. Once this is achieved, one gains confidence in exploring the implementation of FDTD to simulate the EM coverage of more complex and arbitrary tunnel scenarios, whereby frequency-domain analytical efforts tend to be cumbersome and diversified for broad band purposes.

\section{REFERENCES}

[1] A. Taflove, and S. C. Hagness, "Computational Electrodynamic: the Finite-Difference Time-Domain Method", 3rd Edition., Arthec House Publishers, 2005.

[2] S. D. Gedney, "An anisotropic perfectly-matched layer-absorbing medium for the truncation of FD-TD lattices," IEEE Transaction Antennas Propagation., vol. 44, pp. 1630-1639, Dec. 1996.

[3] Y.P. Zhang, Y. Hwang and P.C. Ching "Wide-Band UHF Radio propagation Characteristics in a Tunnel Environment", Wireless Personal Communications 8: 291-299, 1998.

[4] Y. P. Zhang, Y. Hwang, "Characterization of UHF radio propagation channels in tunnel environments for microcellular and personal communications" IEEE Trans. on Vehicular Technology, Vol. 47, No. 1, pp.283-296, February 1998.

[5] S. Kirkpatrick, C. D. Gelatt, M. P. Vecchi (1983-05-13). "Optimization by Simulated Annealing". May 1993. Science. New Series 220 (4598): 671-680

[6] A. Valcarce, G. De La Roche, A. Juttner, D. Lopez, and J. Zhang, "Applying FDTD to the coverage prediction of WiMAX femtocells," in Eurasip Journal of Wireless Communications and Networking, Feb. 2009, Article ID 308606. 
[7] H. C. Rhim and O. Buyukozturk, "Electromagnetic properties of concrete at microwave frequency range," ACI Materials Journal, vol. 95, no. 3, pp. 262-271, 1998.

[8] NVIDIA CUDA (Compute Unified Device Architecture): Programming Guide, Version 3.0. Feb 2010 http://developer.download.nvidia.com/ compute/cuda/3_0/docs/.

[9] T. Rauber and G. Runger, Parallel Programming for multicore and cluster system, Springer-Verlag, 2010.

\section{ACKNOWLEDGEMENT}

This work was supported by CNPq under Covenant 573939/2008-0 (INCT-CSP) and FAPERJ under Fellowship E-26/100.078/2008. 